\title{
A Study on Diabetic Retinopathy Detection Using Image Processing
}

\author{
${ }^{1}$ Manimegalai P, \& ${ }^{2}$ Jayalakshmi P K \\ ${ }_{1,2}$ Dept. of Biomedical Engineering, Karunya Institute of Technology and Sciences (Deemed to be University), \\ Coimbatore, Tamilnadu, India.
}

${ }^{* *}$ Corresponding Author: manimegalaip@karunya.edu.in

Received: 10.01.2021, Revised: 10.02.2021, Accepted: 15.03.2021, Published: 16.04.2021

DOI:

10.53409/mnaa/jcsit/2104

Abstract: This study provides an overview of the Diabetic Retinopathy (DR) detection in human eyes utilizing various preprocessing and segmentation approaches. There are various ways for segmenting the blood veins in the retina. It is possible to determine whether or not the eyes have DR if the retinal nerve fibers have been segmented. This detection is determined by the location of the Retinal nerve fiber layer (RNFL). DR affects nerve fibers with a small total area. If the nerve fiber is large, the eyes are not damaged by DR and thus it is healthy. It is a prominent truth that diabetes has a significant impact on human health and damage every organ, including the eyes. Because the optical nerve is linked to the brain, this DR will influence vision loss in humans. Images of the retinal fundus are frequently utilized to detect and analyze diseases on infected images. Machine learning algorithms struggle to analyze raw retinal fundus images. As a result, a survey is being conducted in this area. In this way, the most current works by diverse authors from across the world are presented in this context. DR is one of the severe infections that may occur in the eyes. According to the WHO - United Nations study, this disease is the second most common among humans. As a result, eye care must be given top priority. This disease is caused by a decrease in the nerve layer of the retina. If the RNFL shrinks, the optic nerve that connects to the brain gets damaged, resulting in vision loss. This document just provides a basic overview of diabetic retinopathy.

Keywords: Diabetic Retinopathy, Nerve Fiber, Retina, Histogram, Pre-processing, Feature Extraction, Segmentation, Image Processing.

\section{INTRODUCTION}

ye diseases are subjected to a variety of infections in the human eye, as well as numerous factors that impact diabetes, blood glucose, and so on. These include retinopathy, cataracts, edemas, and other eye conditions. A variation in the retina's blood veins will cause the capillaries and veins to erupt, resulting in bleeding and the loss of human eyesight. The eyes are the most important organ of human; without them, there would be no vision, and the entire world would be black. The term DR is derived from the Greek word. It is a serious problem that has a direct influence on the human eyes, causing damage to the capillaries and veins since they were very sensitive to brightness because the retina is located at the eye's rear side [1$10]$.

The DR has no symptoms but only causes low vision difficulties, which means that eyesight will be reduced to begin with if the condition is present. When a person becomes diseased with the DR, the veins or capillaries begin to flow blood due to vein eruption. This is caused by diabetes in the circulation, which is extremely close to the retina. Blood vessels damage can result in eyesight loss. As a result of the fluid, the macula swells, resulting in impaired vision. DR develops when retina's blood vessels are damaged by abnormal blood sugar levels. Diabetics are therefore at risk of developing DR, a kind of eye disease. The cornea, iris, retina, nerve fibers, sclera, optic nerve, and other major elements of the eye are represented in Figure 1.

The initial step in detecting is segmenting blood arteries, which can support in classification. The walls of the retina's blood veins have a variety of characteristics, including colour, length, diameter, breadth, tortuosity, branching, and so on [12]. The various components of the human eye, such as the OD, macula, OC, and fovea, impact the blood vessels, which are smaller than the retinal walls [7]. Diabetic patients are more likely to develop Cataracts, Glaucoma, DR and Occlusions in the retinal veins. High blood pressure affects the eyes, resulting in visual loss [34]. 


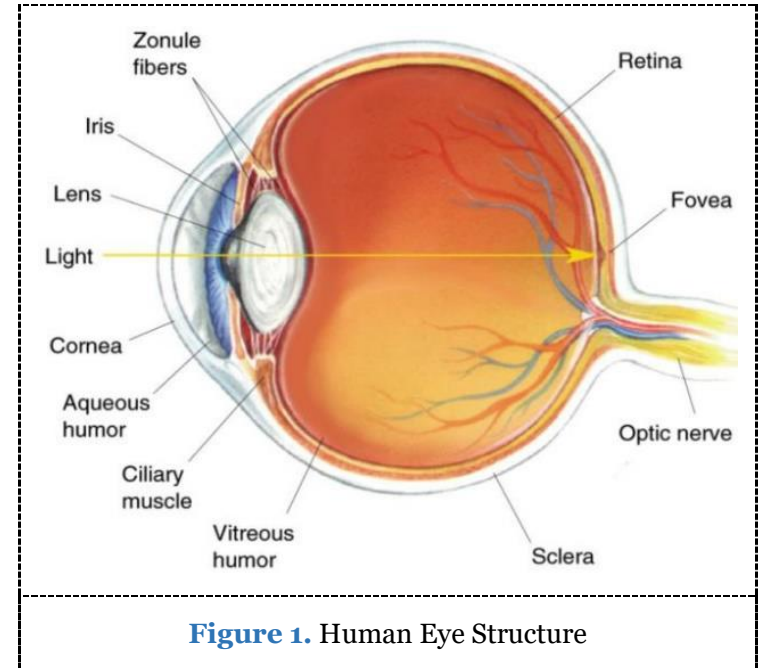

In brief, DB occur due to a variation in the shape of human eye's blood vessel. The retina structure would be a thin, internal covering at the back end of eyes that was extremely light dependent. The damage was induced by an increase in the blood sugar levels, which can injure veins/capillaries. When these veins-capillaries grow and get large, they might cause spills, which causes vision loss in the human eye. Figure 2 depicts the four stages of DR in the form of a slide. Aside from that, it is also described one by one as follows. The four types of DR are described as follows.

- Mild-Initial,

- Moderate-Mid way,

- Severe/non-proliferative pre-final \&

- Proliferative-final stages [21-30].

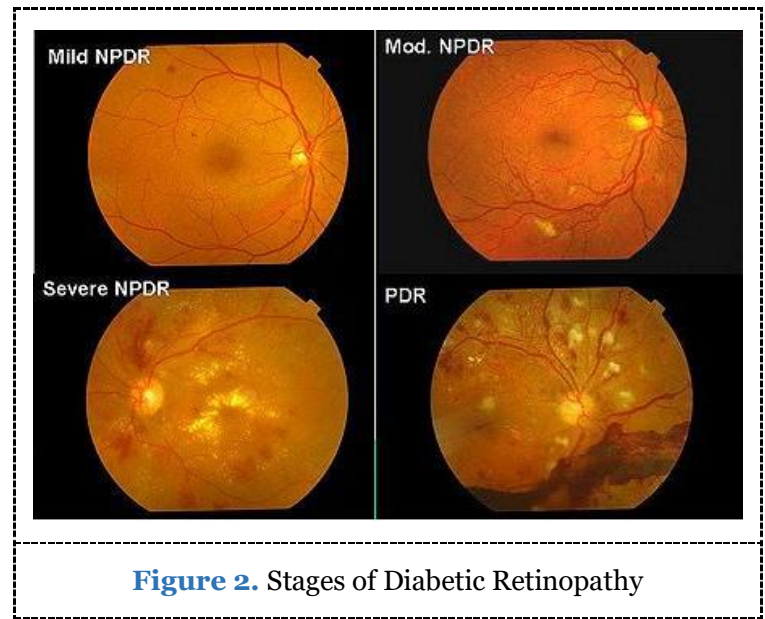

In the initial stage of DR, which is mild or non-proliferate, there would be inflation in the structure of a tiny bubble in some of the blood capillaries or veins present around the retina [3135]. Then, second stage known as medium/moderate non-proliferative retinopathy, some retinal veins are defected as a result of the elevated sugar level, resulting in the occlusion [3640].
In the third stage, severe or non-proliferative DR causes additional blocked veins/capillaries, resulting in many of the retina's zones never receiving adequate blood flow again, resulting in more retinal blockages. As a result, without adequate blood flow, the retina could not produce more or new capillaries/blood veins to replace injured blood vessels [41-45]. The fourth and last stage of DR termed as proliferative retinopathy. This is the advanced stage of the disease, when new blood vessels begin to emerge within retina, i.e., begin to expand, but they will be very fragile and unique (minor in structure). Hence, they can spill blood (leak out the blood), causing diluted vision loss and possibly blindness [46-49].

The study is organized as follows; relevant works concerning the problems addressed in this research article are offered in the introductory section. The next part provides an in-depth overview of the literature review. This follows the shortcomings of the work of many authors. The general technique for detecting diabetic retinopathy in human eyes is detailed in the next part, and finally the survey is concluded.

\section{RELATED WORK}

This section analyses and discusses different studies on diabetic retinopathy and its detection using retinal nerve fibers.

Some innovative approaches for analyzing retina images were proposed in [1]. This study incorporated both the IP and DM principles. [2] discusses a unique technique of supervisory classifications based on blood vessels filtering utilizing Gabor Wavelets Transforms. Novel ways of segmenting blood arteries of the same size, i.e., comparable diameters, were described in [3]. A hybrid technique in [5] was developed for blood vessel identification that relied on feature extraction. [6] describes how to prepare texts in the dictionary patterns for classifying capillaries/blood veins. [7] suggested mathematical models and clustering with k-means for blood capillaries segmentation. An identification technique was presented in [8] on the basis of blood veins classification (areas, shape, and undesirable regional volumes around the retina).

Ant bee-colony optimization and the FCmeans cluster technique were used in [9] to locate small and coarse blood veins, from which the diagnosing the disease can perform. The breakdown of the tree approach was used in [10] to create a rapid methodology for identifying blood vessels in the retina for DR. A GL Spatial Co-relation idea for generating histograms based on the property of the image locally was discussed in [11]. 
The next sections focused on the segmentation techniques employed by different authors to segment the nerve fibers of retina prior to DR identification. A novel algorithm technique detects blood capillary ageing over time and further segments them for disease diagnosis [12]. In [31], an automated technique was proposed for identifying red colours (lesions) in diabetic fundus images to diagnose DR disease. In [30], extensive study was conducted on the segmentation of blood capillaries in images using an integration of center line detection and reconstruction based on morphological characteristics. In [29], the neural networks-based identification of hard exudation in retinal image was discussed. [28] addressed the segmentation of blood vessels in the retina with ANN principles to identify the diseases at the initial stages. An automatic lung module identification method was developed in [27] based on matching profiles and the BP algorithm in ANN.

A quick overview of full background material on the diagnosis of diabetic retinopathy was presented in [24]. It demonstrated how to make a diagnosis of a person suffering from this condition. The research in [23] performed excellent work on identifying the wall of blood capillaries/veins in retina images and tracking them utilizing the ideas of the combination of Gaussian and Kalman filters. The work in [22] conducted extended work on tracing blood veins utilizing extrication of blood vessel characteristics using quick automated techniques of tracing veins and how a direct algorithm may be built for detecting purposes.

A fuzzy vascular track method was proposed in [21] for retina images based on the notion of fuzzy clusters. The work in [20] addressed the identification and quantification of DR disease utilizing digital angiography, which provided good outcomes. The authors' computation relied on the coordinated filtering approach paired with previous knowledge regarding the retinal blood vessels characteristics to differentiate the vessels border boundaries, following vessel's midline, and concentrated on clinically important data. By showing the vessel profile using Gaussian capabilities, they improved vessel width assessments above previous estimates by creating innovative methods. Nonetheless, there was not a lot of variances.

A ridge-based vascular segmentation in the context of a coloured retinal image was discussed in [19]. The model was reliant on how the ridge of image was extricated and corresponded with the blood vessel centrelines. To distinguish between abnormal and normal images, the k-NN classifier was utilized. However, the method's accuracy for higher-order observers was not explored (more than a second). The work in [18] reported the related study of retina blood veins segmentation using innovative approaches on other publicly available database. Nonetheless, the approach produced good outcomes for the selected dataset. It did not offer any data regarding the support of procedures linked to other databases that were available.

The research in [17] performed mathematical modelling and assessment of curvature techniques, which were utilized to segment blood arteries and perform cross curvature assessments. One significant disadvantage was that the noise parameter was not addressed in the research. [13] [14] addressed the differentiation technique of segmenting the retina blood veins and development of the areas, as well as establishing a novel analysis for defining the retina blood capillaries. One limitation of the study was that it only worked for second-order derivative functions and did not fulfil higher-order derivatives.

The work in [33] provided a short summary of imaging approaches for detecting DR disease and its severity consequences, as well as classification strategies. This article evaluated the numerous techniques for DR detection and classification into several phases based on extremities levels, as well as different image DR used for performing the research. The classification difficulties for DR detection, i.e., the multi-class SVM automated identification and analyses of the DR, were explored in [34]. Even while the classifications produced excellent results, it did not satisfy when other classifiers were employed on the same collection of images from standard databases. In [40], a thorough review of current advancements in the diagnosis of DR disease was presented, providing a quick reckoner for DB researchers.

The work in [35] reported research on the DR phases utilizing retinal fundus images and several computer methods. It was utilized for detection; however, the severity of the instances could not be determined. A related group addressed higher demanding spectral problems for recognizing distinct stages of DR [36]. The work in [37] discussed a short analysis of the DR detection utilizing fundus images acquired by the camera. The goal was to create a computerized screening approach that would evaluate automated retinal images for notable feature of non-proliferative DR. Acknowledgment of exudate was calculated using thirty retinal images. For the finding of the HMA, the algorithm obtained 77.5 percent accuracy and 88.7 percent specificity. However, when noises in the fundus retinal images were taken into account, sensitivity and specificity were decreased, which was a serious disadvantage in this study.

The authors of [41] worked on retinal vascular segmentation techniques and presented novel approaches for the same. In general, there are as many approaches and algorithms for segmental 
issues as there were specific cases and situations in the degenerative phases of eye. When everything was discussed and done, segmental ideas are used for medical reasons, and segmental concerns are too specialized for retinal anatomical structures.

A review of diabetic retinopathy and associated literature was conducted. Most studies did not take into account infected images. Limited databases, noise artifacts, the use of typical segmentation techniques, SNR, high computational time and other factors were also not considered.

\section{STEPS TO BE FOLLOWED FOR DETECTING DIABETIC RETINOPATHY}

The first and most important stage is to obtain images from the database or hospitals. Following that, the captured image must be preprocessed. In preprocessing, image conversion, filtering, and denoising can be made. The future challenge in studying retinal-based DR disease is feature extraction, and the following approaches are being employed to do so. After preprocessing, features may be extracted using a variety of approaches. Using image processing techniques, this was the extracting process of the new blood veins from the images. Segmentation, thresholding, smoothing, and morphological operations are the postprocessing processes.

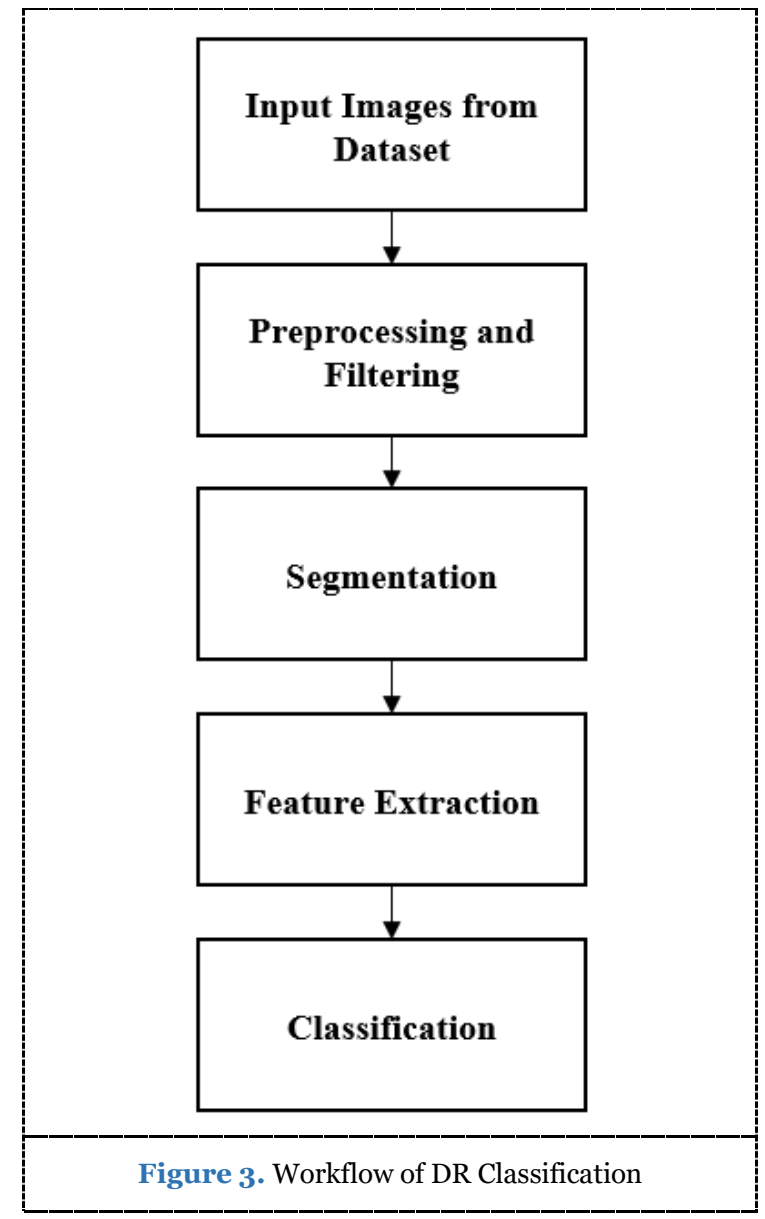

The final step is feature extraction and classification, which is described below. Extraction of features in machine learning, image processing, and digital image processing begins with the basic procedure of evaluated data from retina images and parameters (attributes) designed to be informative and non-repetitive, enabling the following learning and theory processes at times producing better user interpretations. With a decrease in the size of the retinal images, the dimensions of the feature parameters may be determined. After the fundus retinal images have been pre-processed and segmented, there are several techniques for obtaining the features. Some of the approaches include independent components analysis, semantic analyses based on latency, the least square technique based on partial value, PCA, and others. Different classifiers, such as neural networks, machine learning, and deep learning algorithms, can be used to classify based on extracted features. Currently, the majority of studies employ deep learning-based approaches, especially CNN. CNN is a superior alternative for image classification and has produced better image processing outcomes.

\section{CONCLUSION AND FUTURE WORK}

In this study, a quick summary of various principles pertaining to how the DR diseases can be diagnosed at the initial stage was provided in brief, which employed the preprocessing, segmentation, and feature extraction techniques. In this survey, many researchers who worked in this subject and made significant contributions to diabetic retinopathy were given in the form of a comprehensive literature assessment, along with their advantages and limitations. The information provided in this study was simply the work made by several authors, so that researchers can be aware of recent advancements in the work made on DR and its relevant aspects and also additionally refine the research with more information, defining the research problems while taking into account the shortcomings of the existing researches.

This survey examined the essential needs of numerous disease eradication schemes, evaluated suggested solutions for these issues, and emphasized the varied detection and performance strengths, as well as disadvantages and shortcomings, of the work done by various researchers. The major contribution of this work was to inspire additional researchers to propose and enhance possible ideas for different forms of DR disease detection utilizing various techniques. Finally, this study provides a brief overview of diabetic retinopathy disease and research findings. This study therefore provided a brief overview of the scientific effort on identifying the world's second most serious disease, which may be identified 
utilizing innovative pre-processing and segmentation approaches.

\author{
ETHICS APPROVAL AND CONSENT TO \\ PARTICIPATE \\ Not applicable.
}

HUMAN AND ANIMAL RIGHTS

No animals/humans were used for studies that are basis of this research.

\section{CONSENT FOR PUBLICATION \\ Not applicable.}

AVAILABILITY OF DATA AND MATERIALS

The authors confirm that the data supporting the findings of this research are available within the article.

\section{FUNDING}

None.

\section{CONFLICT OF INTEREST}

The authors declare no conflict of interest, financial or otherwise.

\section{ACKNOWLEDGEMENTS}

The authors would like to thank their present employer for providing support while carrying out this research work.

\section{REFERENCES}

[1]. Ramani G, Balasubramanian L. Retinal blood vessels segmentations employing images processing and data mining technique for computerized retinal images analyses. J. Biocybernetic Biomed. Eng., 2016, 36(1), 102-118.

[2]. Songyuan T, Lin T, Yang J, Fan J, Ai D, Wang Y. Retinal Vessels Segmentations using Supervised Classifications based on Multiscale vessels filtering and Gabor Wavelets. J. Med. Imaging \& Health Infor., 2015, 5(7), 1571-1574.

[3]. Allan J F, Undrill PE, Cree MJ, Olson JA, McHardy KC, Sharp PF, Forrester JV. A comparisons of computers-based classifications method applied to the detections of microaneurysm in ophthalmic fluorescein angiogram. Comput. Biol. Med., 1998, 28(3), 225-238.

[4]. Usman A M and S A Khan. Multilayer thresholding- based blood vessels segmentations for screenings of diabetic retinopathy. Eng. Comput., 2013, 29(2), 165-73.

[5]. Land, M. F, The human eyes: Structures and functions. Nat. Medic., 1999, 5(11), 1229-1229.

[6]. Zhang L, Mark F, Wenjia W. Retinal vessels segmentations using multi-scale text on derived from Keypoint. J. Comput. Med. Imaging Graph., 2015, 45, 47-56.

[7]. Sinthanayothin C. Automated localizations of the optical discs, fovea \& retinal blood vessel from digital color fundus image. Br. J. Ophthalmal., 1999, 83(8), 902-910.

[8]. Sinthanayothin C, Boyce JF, Williamson TH, Cook HL, Mensah E, Lal S, Usher D. Automated detections of diabetic retinopathy on digital fundus image. Diabetic. Med., 2002, 19(2), 105-112.
[9]. Koen V, Vos FM, Lemij HG, Vossepoel AM. A model-based method for retinal blood vessels detections. Comput. Biol. Med., 2004, 34(3), 209-219.

[10]. Subhasis C, Chatterjee S, Katz N, Nelson M, Goldbaum M. Detections of blood vessel in retinal image using twodimensional matched filter. IEEE Trans. Med. Imaging, 1989, 8(3), 263-269.

[11]. Thitiporn C, Guoliang F. An efficient algorithm for extractions of anatomical structure in retinal image. International Conference on Image Processing, 2003, pp. 11931196.

[12]. Hoover A. D, Valentina K, and Michael G. Locating blood vessel in retinal image by piecewise threshold probing of matched filters responses. IEEE Trans. Med. Imaging, 2000, 19(3), 203-211.

[13]. Martinez-Perez ME, Hughes AD, Stanton AV, Thom SA, Bharath AA, Parker KH. Segmentations of retinal blood vessel based on the second directional derivatives \& regions growing. International Conference on Image Processing, 1999, pp. 173176.

[14]. Martinez-Perez ME, Hughes AD, Stanton AV, Thom SA, Bharath AA, Parker KH. Scale-space analysis for the characterization of retinal blood vessels. Medical images computing and computer-assisted interventions, 1999, pp. 9097.

[15]. Yiming W and Samuel C.L. A fast method for automated detections of blood vessel in retinal image. Conferences Records of the 31st Asilomar Conferences on Signal, System and Computer, 1998, pp.1700-1704.

[16]. Xiaoyi J, Daniel M. Adaptive local thresholding by verifications based multi threshold probe with applications to vessels detections in retinal image. IEEE PAMI., 2003, 254(1), 131-137.

[17]. Frederic Z, Klein JC. Segmentations of vessels-like pattern using mathematical morphology \& curvatures evaluations. IEEE Trans. Image Process., 2001, 11(7), 1111-1119.

[18]. Meindert N, Staala J, Ginnekena VB, Looga M, Abramoff MD. Comparative study of retinal vessels segmentations method on a new publicly available database. Medical imaging 2004: Image Processing, 2004, 5370, pp. 1-9.

[19]. Joes S, Abràmoff MD, Niemeijer M, Viergever MA, Van Ginneken B. Ridges-based vessels segmentations in colour image of the retina. IEEE Trans. Med. Imaging, 2004, 23(4), 501-509.

[20]. Liang Z, Rzeszotarski MS, Singerman LJ, Chokreff JM. The detections and quantifications of retinopathy using digital angiogram. IEEE Trans. Med. Imaging, 1994, 13(4), 619-626.

[21]. Yannis T and Stavros M P. A fuzzy vessels tracking algorithms for retinal image based on fuzzy clustering. IEEE Trans. Med. Imaging, 1998, 17(2), 263-273.

[22]. Ali C, Shen H, Turner JN, Tanenbaum HL, Roysam B. Rapid automated tracings and features extractions from retinal fundus image using direct exploratory algorithm. IEEE Trans. Inf. Technol. Biomed., 1999, 3(2), 125-138.

[23]. O. Chutatape, Liu Z, Shankar M K. Retinal blood vessels detections and tracking by matched Gaussians and Kalman filter. Proceedings of the 20th Annual International Conference of the IEEE Engineering in Medicine and Biology Society., 1998, pp. 3144-3149.

[24]. Vivian S L, Kingsley RM, Lee ET, Lu M, Russell D, Asal NR Bradford RH Jr, Wilkinson CP. The diagnoses of diabetic retinopathy, Ophthalmoscopy vs fundus photography. J. Ophthalmology, 1993, 100, 1504-1512.

[25]. Research Section. Digital Retinal Image for Vessel Extraction (DRIVE) Database. Univ. Med. Center Utrecht, Image Sci. Inst., Utrecht, The Netherlands, 2000. 
[26]. Toulson DL, James F B. Segmentations of MR image using neural net. BMVC91. Springer, 1991, pp. 284-292.

[27]. Shih-Chung L, Freedman MT, Lin JS, Mun SK. Automatic lungs nodules detections using profiles matching and backpropagations neural networks technique. J. Digital Imaging, 1993, 6, 48-54.

[28]. Mann K S, Sukhpreet K. Segmentations of retinal blood vessel using artificial neural network for early detections of diabetic retinopathy. AIP Conference Proceedings, 2017, 1836(1), pp. 1-9.

[29]. María G, Sánchez CI, López MI, Abásolo D, Hornero R. Neural networks-based detections of hard exudate in retinal image. Comput Method Program Biomed., 2009, 93, 9-19.

[30]. Ana M. M, Aurelio C. Segmentations of retinal blood vessel by combining the detections of centerline \& morphological reconstructions. IEEE Trans. Med. Imaging, 2006, 25(9), 12001213.

[31]. Michael L, Godt J, Larsen N, Lund-Andersen H, Sjølie AK, Agardh E, Kalm H, Grunkin M, Owens DR. Automated detections of fundus photographical red lesion in diabetics retinopathy. Investig. Ophthalmol. Vis. Sci., 2003, 44(2), 761-766.

[32]. Adam H, Michael G. Locating the optical nerves in retinal images using the fuzzy convergences of the blood vessel. IEEE Trans. Med. Imaging, 2003, 22(8), 951-958.

[33]. Paranjpe M J, Kakattkar MN. A Review of method for diabetic retinopathy detections and severity classifications. IJRET, 2014, 3(3), pp. 619-624.

[34]. Adarsh P, Jeyakumari D. Multiclass SVM-Based Automated Diagnoses of Diabetics Retinopathy. International Conference on Communication and Signal Processing., 2013, pp.3-5.

[35]. Acharya U.R, Lim CM, Ng EY, Chee C, Tamura T. Computers-based detections of diabetic retinopathy stage using digital fundus image. J. Eng. Med., 2009, 223, 545-553.

[36]. Acharya U.R, Chua CK, Ng EY, Yu W, Chee C. Applications of higher-order spectral for the identifications of diabetic retinopathy stage. J. Med. Syst, 2008, 32(6), 481-488.

[37]. Sinthanayothin C, Boyce JF, Williamson TH, Cook HL Mensah E, Lal S, Usher D. Automated detections of diabetic retinopathy on digital fundus image. J. Diabetic. Med., 2002, 19, 105-112.

[38]. Upendhra K. Significant Enhancements of Segmentations Efficiency of the Retinal Image Using Textures-Based Gabors Filters Approach Followed by Optimizations Algorithms Ophthalmology: Breakthrough in Researches and Practices, IGI, 2018, pp. 53-68.

[39]. Gehhad H, Aboul EH. A Review of Vessels Segmentations Methodologies and Algorithm: Comprehensive Review. Handbooks of Research on Machine Learning Innovation and Trend, 2017, pp. 187-203.

[40]. Javerria A, Muhamad S, Musarat Y. A Review on Recent Development for Detections of Diabetics Retinopathy. Scientifica, 2016.

[41]. Jasem A, Khaled E, Abdelrahman E. Retinal Vessel Segmentations Technique and Algorithm: A Survey. Appl. Sci., 2018, 8(156), 1-31.

[42]. Michael J. F. Microvascular and Macrovascular Complication of Diabetes. Clin. Diabet., 2008, 27(2), 77-82.

[43]. Ronald K, Klein BE, Moss SE, Wong TY. Retinal vessels calibers and microvascular and macrovascular diseases in types 2 diabetes: XXI: the Wisconsin Epidemiologic Study of Diabetics Retinopathy. Ophthalmol., 2007, 14(10), 1884-1893.

[44]. Rezatofigi SH, Rodaki A, Noubhari HA. An enhanced segmentations of blood vessel in retinal image using contourlets.
International Conference of the IEEE Engineering in Medicine and Biology Society, 2008, pp. 3530-3533.

[45]. Peng F, Pan Y, Wei B, Jin W, Mi D. Enhancing retinal images by the Contourlets transforms. Patt. Recogn. Lett., 2007, $28,516-523$.

[46]. Qing ZH. Segmentations of blood vessel in retinal image using 2-D entropies of grey levels-gradients co-occurrences matrix. IEEE, 2004, pp.508-513.

[47]. Susanta M, Bhabatosh C. Hues preserving color images enhancements using multi-scale morphology. ICVGIP, 2002.

[48]. Stephanie G M. A theory for multi-resolutions signals decompositions the wavelets representations. IEEE PAMI., 1989, 11(7), 674-689.

[49]. Mohammed AR, Munib, Mohammed A. An improved matched filters for blood vessels detections of digital retinal image. Comput. Biol. Med., 2007, 37, 262-267. 